https://doi.org/10.36396/MS.2019.14.03.003

\title{
Роль шкалы SYNTAX Score II в принятия решений «сердечной командой» о методе лечения пациентов со сложными поражениями коронарных артерий. Обзор литературы
}

\author{
Б.Г. ААЕКЯН ${ }^{1}$, Н.Г. КАРАПЕТЯН ${ }^{1}$, В.В. КРАВЧЕНКО', А.Ш. РЕВИШВИАИ' \\ 1 ФГБУ «Национальный медицинский исследовательский центр хирургии им. А.В. Вишневского» Минздрава России, Москва, Россия
}

\begin{abstract}
С момента своего внедрения в клиническую практику чрескожные коронарные вмешательства (ЧКВ) постепенно стали методом выбора при лечении пациентов с ^окальными и несложными поражениями венечных артерий, резистентных к оптимальной медикаментозной терапии. Несмотря на это, использование ЧКВ мяя лечения пациентов с тяжелыми формами поражений коронарных артерий является предметом разночтений и споров и в настояшее время. В эпоху стентов с лекарственным антипролиферативным покрытием одним из наиболее важных и значимых исследований эффективности ЧКВ и коронарного шунтирования является, бесспорно, исследование SYNTAX (Synergy between Percutaneous Coronary Intervention with TAXUS and Cardiac Surgery - согласованность ЧКВ с имплантацией стентов TAXUS и кардиохирургией). Полученные в ходе Аанного исследования результаты легли в основу рекомендаций по реваскуляризации миокарда Европейского обшества кардиологов (EОК) 2014 и 2018 гг. ОАнако на Аанный момент имеются крайне важные и принципиальные несоответствия межАу современной клинической практикой и рандомизированным исследованием SYNTAX. Наиболее сушественным недостатком шкалы риска SYNTAX считается отсутствие индивидуализированного подхода при принятии решения о выборе метода реваскуляризации миокарда в силу отсутствия в ней клинических переменных. В Аанной статье будет рассмотрена новая шкала риска SYNTAX Score II (SS II) как более точный инструмент принятия решения в работе «сердечной команды».
\end{abstract}

Кцючевые слова: реваскуляризация миокарда, коронарное шунтирование, чрескожные коронарные вмешательства, многососудистое поражение коронарных артерий, сердечная команда, SYNTAX Score, SYNTAX Score II

\section{The role of the SYNTAX Score II scale in the decision making of the "heart team" about the treatment of patients with complex coronary artery lesions: a literature review}

\author{
ALEKYAN B.G. ${ }^{1}$, KARAPETYAN N.G. ${ }^{1}$, KRAVCHENKO V.V. ${ }^{1}$, REVISHVILI A.SH. ${ }^{1}$ \\ ${ }^{1}$ Vishnevskiy Institute of Surgery, Moscow, 117997, Russian Federation
}

\begin{abstract}
Since its introduction into clinical practice, percutaneous coronary interventions $(\mathrm{PCI})$ have gradually become the method of choice for treating patients with local and uncomplicated lesions of the coronary arteries that are resistant to optimal medicamental therapy. Despite this, the use of $\mathrm{PCI}$ for the treatment of patients with coronary artery disease carts is a matter of discrepancies and disputes even today. In the era of drug-elongated antiproliferative stents, one of the most important and significant studies of the efficacy of PCI and coronary shunting (CS) is undoubtedly the SYNTAX study (Synergy between TAXUS and Cardiac Surgery implantation of TAXUS and Cardiac Surgery). The results of this study formed the basis for the latest recommendations on myocardial revascularization of the European Society of Cardiology (EOC) 2014 and beyond 2018. However, at the moment there are extremely important and fundamental inconsistencies between modern clinical practice and the randomized SYNTAX study. The most significant drawback of the SYNTAX risk scale is the lack of an individualized approach when deciding on the choice of myocardial revascularization method due to the absence of clinical variables in it. This article will look at the new Syntax Score II risk scale as a more accurate decision-making tool in the work of the "heart team".
\end{abstract}

Key words: myocardial revascularization, $C A B G, P C l$, multivessel coronary artery, Heart team

\section{Сведения об авторах:}

Алекян Баграт Гегамович - акаА. РАН, проф., А. М. н., руководитель Шентра рентгенэндоваскулярной хирургии НМИЦ им. А.В. Вишневского; https://orcid.org/0000-0001-6509-566X

Карапетян Нарек Григорьевич - ст. научный сотрудник Шентра рентгенэндоваскулярной хирургии НМИЦ им. А.В. Вишневского, к. м. н., рентгенэндоваскулярный хирург; https://orcid.org/0000-0002-7623-8635, ngkarapetyan@gmail.сот (автор, ответственный за переписку)

Кравченко Вера Викторовна - аспирант, рентгенэндоваскулярный хирург

Ревишвики Амиран Шотаевич - акаА. РАН, проф., А. м. Н., Аиректор НМИЦ им. А.В. Вишневского 
С момента своего внедрения в клиническую практику чрескожные коронарные вмешательства (ЧКВ) постепенностали ме коро ными поражениями коронарных артерий, резистентных оп, эо, Примененио ЧКВ для ленени пациентов со сложными формами поражений коронарных артерий является предметом разнотении и споров по настоящее время. Как известно из истории развития эндоваскулярной хирургии ишемической болезни сердца (ИБС), после первых удачных операций чрескожная реваскуляризация миокарда прошла долгий, непростой, но крайне плодотворный путь развития от первого баллонного катетера, изготовленного на кухонном столе A. Gruntzig, до современных стенто с лекарственным покрытием четвертого поколения и методов внутрисосудистой визуализации и физиологической оценки коронарного кровотока. Естественно, все новые шаги в развитии эндоваскулярных технологий закреплялись многочисленными успешными результатами многоцентровых рандомизированных клинических исследований (РКИ). На ранних стадиях развития технологий реваскуляризации миокарда основной целью проведенных РКИ было сравнение результатов реваскуляризации миокарда с оптимальной медикаментозной терапией. Далее, уже с появлением различных технологий ЧКВ, РКИ проводили большей частью с целью сравнения эффективности коронарного шунтирования (КШ) и ЧКВ (рис. 1) В эпоху стентов с лекарственным антипролиферативным покрытием одним из наиболее важных и значимых исследований является РКИ SYNTAX (Synergy between Percutaneous Coronary Intervention with TAXUS and Cardiac Surgery - согласованность ЧКВ с имплантацией стентов TAXUS и кардиохирургией) [2]. Полученные в ходе данного исследования результаты легли в основу рекомендаций по реваскуляризации миокарда Европейского общества кардиологов (ЕОК) 2014 [3] и 2018 [4] гг. Также результать SYNTAX дали толчок таким крупным клиническим исследованиям, как EXCEL, SYNTAX II, NOBLE, FREEDOM и т.д. [5-8].

В результате исследования SYNTAX было доказано, то как в ближайшем, так и в отдаленном послеоперационном периоде результаты КШ у большинства пациентов со сложными трехсосудистыми (средний и высокий риск, SYNTAX Score - SS >23) и стволовыми (высокого риска, SS>33) поражениями коронарных артерий достоверно лучше, чем при ЧКВ, за счет меньшей частоты наступления конечных точек (смерть, острый инфаркт миокарда, повторная реваскуляризация, острое нарушение мозгового кровообращения). Лишь у пациентов, имеющих низкии риск SS $(<22)$ при трехсосудистом поражении и средни и низкий риск при Своловом поражении, результаты ЧКв и КШ сопоставимы, что позволяет одавать предпочтение ыполнению ЧКВ в вмешательства [9-10].

Однако в настоящее время имеются крайне важные и принципиальные несоответствия между современно клинической практикой, основанной на публикациях последних лет, и исследованием SYNTAX. Так, первые пациенты были официально включены в исследование SYNTAX с 20 июня 2005 г., а финальные пятилетние результаты были опубликованы в 2012 г. [11]. Таким образом, между началом исследования SYNTAX и публикацией рекомендаци к реваскуляризации миокарда ЕОК прошло 9 лет. На протяжении этого периода времени с параллельным развитием новых технологий и материалов в эндоваскулярной хирурии в научной литературе появилось большое количество публикаций, указывающих на заметное улучшение результатов ЧКВ у пациентов со сложными формами поражения коронарных артерий. Иными словами, мы сегодня пользуемся шкалой риска ЧКВ для принятия решения по выбору стратегии реваскуляризации миокарда, которая была создана 6 лет назад на основании использования стентов с лекарственным покрытием первого поколения (2006 г.), но экстраполируем эти результаты на реалии сегодняшнего дня, когда имеются в наличии современные инструменты и намного более совершенные технологии. Указанный фак заставляет задаваться важными вопросами: соответствую ли результаты исследования SYNTAX и сама шкала риска

Рис. 1. С 1964 г., после первых операций КШ, и Аалее с появлением технологии ЧКВ в 1977 г. в мире постоянно проводияис крупные РКИ, сравниваюшие различные стратегии лечения больных с ИБС

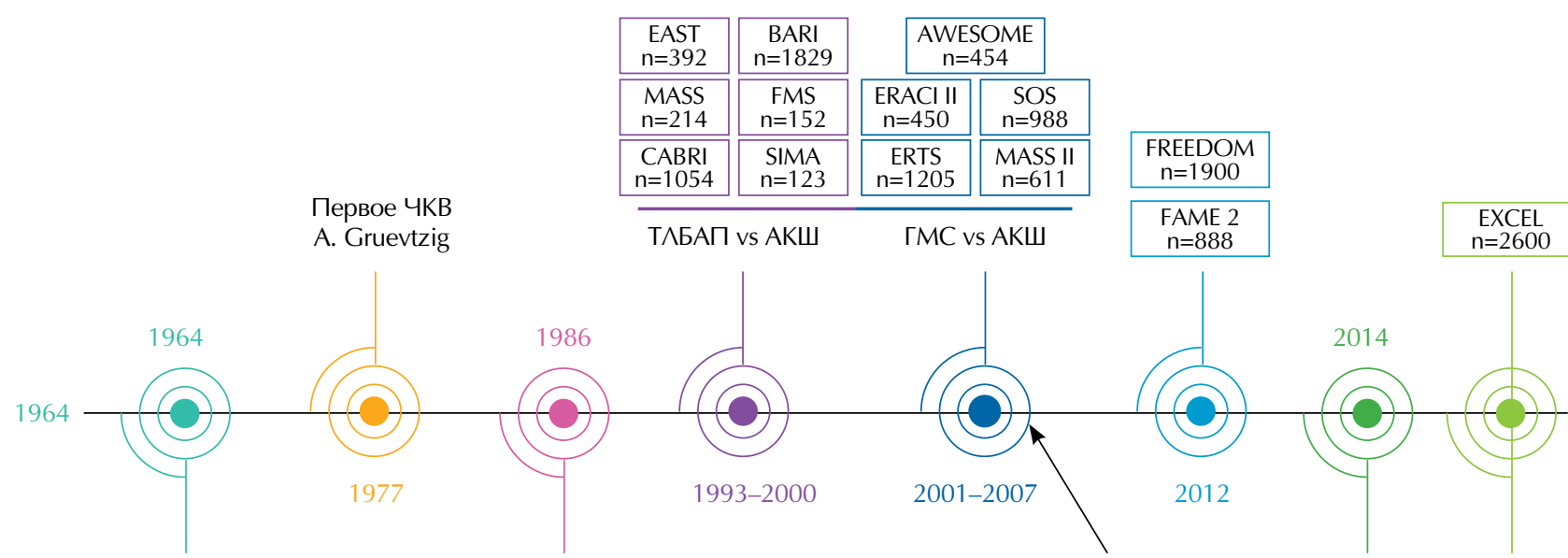

Рис. 2. Кривые Каплана - Мейера цяя пациентов из группы ЧКВ в регистре CREDO-Kyoto, которые поцчеркивают

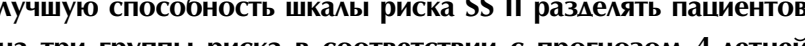

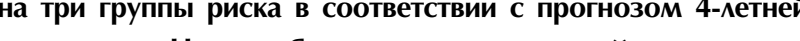
смертности. На оси абсиисс, соответствуюшей прошелшему времени после вмешательства, пашиенты согласно шкале SS (A) расположены достаточно близко Аруг к Аругу, в то время қак SS II (B) pрзздекет больных на три труппы риска боле четко [20]
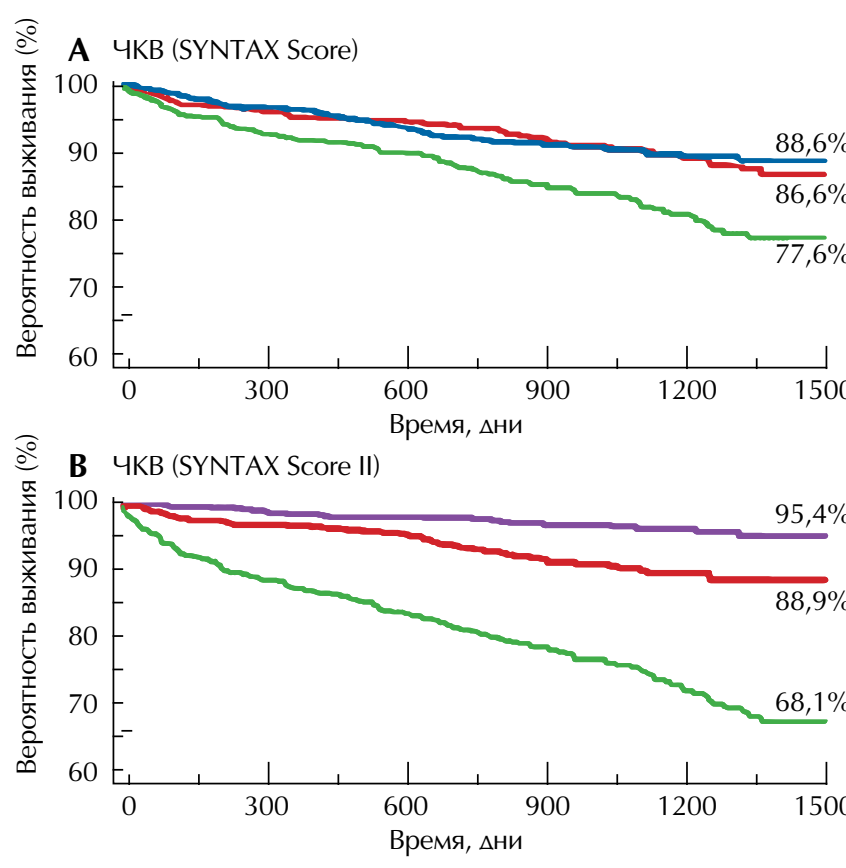

— низкий риск — среАний риск — высокий риск

SS настоящему положению дел и позволяют ли объективно выбрать правильную стратегию реваскуляризации у пациентов со сложными формами поражения коронарн артерий с учетом радикального улучшения инструменто и материалов эндоваскулярной хирургии? Всегда ли является эффективной работа «сердечной команды» и выбор стратегии реваскуляризации, основанный только на анатомии поражения коронарных артерий? Не сужает ли потенциальные возможности ЧКВ калькулятор SS, разработанны раньше, но используемый в современных условиях?

Но для начала необходимо подчеркнуть, какие именно новейшие достижения эндоваскулярной хирургии могуг влиять на результаты реваскуляризации миокарда у пациентов при ЧКВ, которые не были или были недостаточно полно использованы в исследовании SYNTAX. Наиболее важными отличиями являются новейшие стенты с лекарственным покрытием, возможности выполнения инвафракционного резерва кровотока (ФРК) и моментального резерва кровотока (МРК), внутрисосудистые метолы визуализашии: внутрисосудистое ультразвуковое исслегование (ВСУзИ) и оптическая когерентная томография (ОКТ) а также новые инструменты реканализашии хронических окклюзий коронарных артерий (ХОКА) и разработка новой шжалы риска - SS II, позволяюшей оценить пашиента не олько анатомически, но и в совокупности с сопутствуюими клиническими данными и тем самым сформировать инливидуальный прогноз перед выполнением ЧКВ и КШ Тким образом, вышеперечисленные факторы как по отельности, так, возможно, и в совокупности могут влиять а результаты выполнения ЧКВ у пациентов со сложными формами поражения коронарных артерий, что можег и должно расширить границы показаний к выполнению ЧКВ. Это становится особенно актуально и для нашей страны, так как за последние годы в Российской Федерации отмечается резкое увеличение числа выполненных эндовас кулярных операций. Так, если в 2008 г. в РФ выполнялос В 32 тыс. ЧКВ, то уже в 2017 г. - более 200 тыс. [12].

В литературе имеется большое количество публикаций, посвященных стентам последнего поколения, ФРК, ВСУзИ, ОКТ и пр. Но, на наш взгляд, среди достижений, описанных выше, для работы «сердечной команды» наиболее важным является эффективная шкала риска, которая позволяет более полно и точно оценить риски для каждог конкретного пациента, позволяя сформулировать индивидуализированный прогноз. Таким инструментом нам вися новая шкала риска SS II.

В 1981 г. Leaman и соавт. разработали «коронарную систему подсчета баллов, позволяюшую оценить тяжест поражения коронарных артерий. Задачей системы было группы КШ в регистре СREDO-Куото [20]. Обрашает на себя внимание, что шкала SS (C) располагает больных практически параммельно на временной оси, в то время ка при использовании шкалы SS II (D) у больных после КШ имее ессто четкое распрехеление больных по группам риска
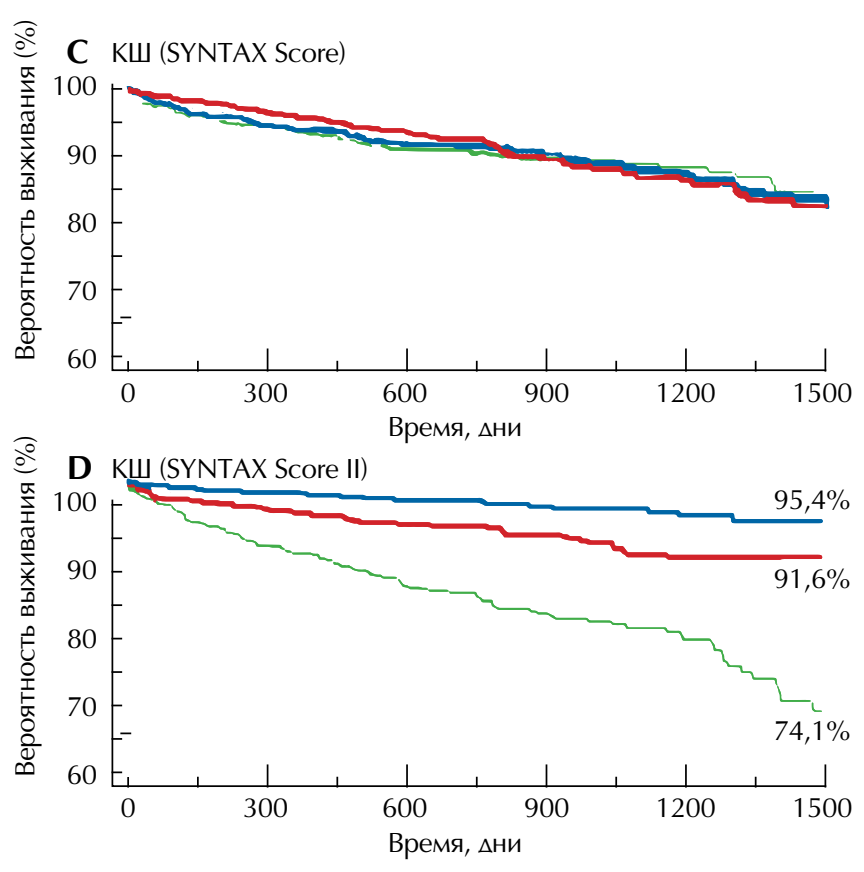

— низкий риск — средний риск — высокий риск 
и значимости данного сосуда для кровоснабжения миокара левого желудочка (ЛЖ). Это была одна из первых рабо по данной проб

Вместе с тем интересно напомнить, что задолго до этоо, в 1976 г., в советском журнале «Кардиология» [13] вышла публикация Ю.С. Петросяна и Д.Г. Иоселиани, которые разработали методику суммарной оценки состояния коронарного русла у больных ИБС. Это была первая попытка прогнозирования результатов вмешательства на основании изучения коронарограмм пациентов. Более

Исследование SYNTAX было посвящено сравнению
боступной нам литературе найти не удалось. результатов прямой реваскуляризации миокарда с высокой частотой использования артериальных шунтов и стентирования коронарных артерий стентами с лекарственным покрытием TAXUS (первого поколения) у групп высокого риска со стенозом ствола левой коронарной артерии (ЛКА) и трехсосудистыми поражениями коронарных артерий В созданной после выполнения исследования шкале риска SS проводится анализ анатомической локализации поражений: бифуркационное поражение, устьевое, тотальная окклюзия, тромбоз, кальцифицированность, поражение мелких сосудов на основании данных коронарной ангиографии [14]. Шкала риска SS является независимым предиктором отдаленных больших кардиальных и цереброваскулярных собылй в групте пациентов, подверншихся ЧКВ (но не КШ). Она облегчает работу «сердечной команды» и выбор опимальног метода реваскуляризации, так как позволяет выявить пациентов с высоким риском развития неблагоприятных событий после возможного ЧКВ. Шкала SS включена для использования как в европейские, так и в американские рекомендации по реваскуляризации миокарда. В них также указано, что для выбора наиболе оптимальной тактики реваскуляризации миокарда при проведении консилиума «сердечной команды», в состав которого входят кардиолог, кардиохирург и рентгенэндоваскулярный хирург, клинические данные пашиента также должны быть приняты во внимание. Однако отсутствие клинических показателей в самой шкале SS является ее безусловным недостатком.

Так как для прогнозирования отдаленных результатов имеет большое значение наличие сопутствуюших заболеваний, потребовалось создание новой шкалы расчета риска - SS II [15]. Шкала риска SS II включает в себя две анатомические переменные: рассчитанный ранее показатель SS и наличие поражения ствола ЛКА (то есть если имеется поражение ствола ЛКА, то при расчете риска по шкале SS II поражение ствола ЛКА указывается повторно). Также шкала содержит 6 клинических переменных (возраст, пол, скорость клубочковой фильтрации, фракция выброса ЛЖ (ФВ ЛЖ), наличие хронической обструктивной болезни легких (ХОБЛ), наличие поражения периферических артерий). Особенностьо шкалы риска SS II является также возможность прогнозирования вероятности 4-летней смертножкак в случае выполнения КШ, так и при выполнении ЧКВ (в отличие от своего предшественника, который не може

SS II благодаря наличию клинических показателей считаболее достоверной, чем SS, в плане прогнозирования . В настоящее время ведутся работы с более широким применением данной шкалы в клинической практике. Тем не меколичество публикаций, посвященных эффективности лы SS II, невелико.

Наиболее тяжелая группа больных с ИБС - это те, которые имео сочекание поражения коронарных артерии с такими хроническими заболеваниями, как ХОБЛ, хроническая сердечная недостаточность (ХСН), хроническая болезнь почек (ХБПП) и т.д. Краеугольным камнем для данно когорты больных является точный выбор эффективной безопасной стратегии реваскуляризации миокарда с уче-

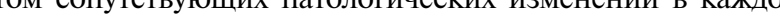
қонолем случае. Только анатомическая оценка паци ентов с наличием или отсутствием тяжелой хронической палологии не является корректой. Как показываеп реальная клиническая практика, далеко не каждому пациенту с низким SS можно выполнять ЧКВ и не всем пациентам со средним и высоким SS можно успешно выполнить КШ Сопугсвующие клинические факторы, такие как низкая ФВ ЛЖ, уровень креатинина, ХОБЛ и другие, могут ухудшить или вообще сделать невозможным выполнение КШ пациентов с высоким SS. Приведем пример: два пациента одного возраста и значением SS=34 не могут быть клинически одинаковыми, если у одного имеется ХОБЛ и тяжелос поражение артерий нижних конечностей или внутренних сонных артерии, а у другого нег сонугсыующих заболеваний. С одной стороны, обоим пациентам выполнение ЧК не показано, согласно рекомендациям ЕОК, и необходим отдавать предпочтение КШ. Тем не менее выполнение КШ у первого пациента с тяжелой сопутствующей патологией, скорее всего, будет невозможно.

Шкала SS II была разработана при ретроспективном SYNTAX ( $\mathrm{n}=1800)$, и клинические переменные, входящие в состав нового калькулятора и влияющие на результаты реваскуляризации, также были определены при одном и том же рандомизированном исследовании, что и ее предшественник.

V. Farooq и соавт. [15] разработали данную шкалу путем статистического анализа взаимодействия клинических переменных со шкалой риска SS. Базовые характеристики пациентов, которые имели сильные корреляции с 4-летнеи да (КШ или ЧКВ), были добавлены к анатомической шкале SYNTAX. Для каждого пациента были сделаны сравнения прогнозов смертности в течение 4 лет между КШ и ЧКВ. Далее оценка точности новой шкалы была проведена в международном реестре DELTA $(\mathrm{n}=2891)$, который включал па циентов с незашищенным стволом ЛКА (изолированный или в сочетании с двух-, трехсосудистым поражением коронарных артерий),которым было выполнено КШ или ЧКВ. Среди всей когорты пациентов у 744 (26\%) имелось трехсосудистое поржене ве казатель SS $\geq 33$ имел место у 902 (30\%) [18]. Показатель SS I ре сказ пациентов, которым было выполнено КШ, и теми, которые голверглись ЧКВ. Наличие сахарного диабета не оказырало

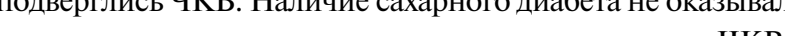
КШ (pКШ (p-interaction 0,67). Была создана номограмма, которая позволила нолуниь тоное индивидуальное предсказание о 4-летнеи смер носп у пақиенов, которым было решено выполнить реваскуляризацию миокарда. Было доказано, что на одаленные пронноз оказызают влияние не только значение SS, но и клй секие перем ло к возрастанию интереса использования шкалы SS II в работе «есда не только принимая во внимание анатомические факторы.

Исследование SS II представляет собой многоцентровое исследование, которое изучало влияние современной стратегии ЧКВ на клинические исходы у пациентов с трехсосудистым поражением коронарных артерий в 22 центрах 4 европейских стран [16]. J. Escaned и соавт. исследовали последние технические инновации в эндоваскулярной хирургии ИБС и их влияние на результаты реваскуляризации миокарда у пационов с тяжелым поражением коронарных артерий. Также определяли эффективность лечения этих больных при принятии решений о стратегии реваскуляризации «сердечной командой», основанной на шкале риска SS II [16]. Стратегия SS II включает в себя следующие важные компоненты: принятие решений «сердечной командой» с использованием шкалы риска SSII, реваскуляризацию с учетом результатов исследования коронарной физиолоОиии значимости поражений артерий (измерение ФРК или МРК), имплантацию стентов с лекарственным рассасываюшимся полимерным покрытием последнего поколения, применение ВСУЗИ, современных методик реканализации хронических окклюзий коронарных артерий и медикаментозную терапию в соответствии c современными рекомендациями. Частота основных неблагоприятных сердечных и цереброваскулярных событий (МАССЕ) в течение одного тода из исследования SS II сравнивали с когортой ЧКВ из ранее выполненного исследования SYNTAX (то есть исторический контроль) Больные были включены в исследование для выполнения ЧКВ при наличии равновероятной 4-летней смертности между КШ и ЧКВ (то есть больные, которым можно рекомендовать как ЧКВ, так и КШ согласно шкале SS II). В обшей сложности 708 пациентов были обследованы обсуждены «сердечной командой» в рамках этого исследования, 454 из которых было решено выполнить ЧКВ. В течение года результаты лечения у больных, которым выполнено ЧКВ с использованием шкалы риска SS II, были лучше, чем у пациентов с ЧКВ из исследования SYNTAX (исторического контроля) - MACCE SS II 10,6\% проти SS $17,4 \%$, OP $0,58,95 \%$ ДИ $0,39-0,85, p=0,006$. Эта разница была обусловлена значительным снижением частоты инфарктов миокарда (ИМ), ОР $0,27,95 \%$ ДИ $0,11-0,70$ $\mathrm{p}=0,007$, и повторной реваскуляризации, ОР $0,57,95 \%$ ди $0,37-0,9, \mathrm{p}=0,015$. По (ОР $0,69,95 \%$ дИ $0,27-1,73, \mathrm{p}=0,43$ ) и инсульта (ОР 0,69 , $95 \%$ дИ $0,10-4,89, \mathrm{p}=0,71$ ) были аналогичными Частота спешифического тромбоза стента была значительно ниже группе из исследования SS II (OP 0,26, 95\% ДИ 0,07-0,97, $\mathrm{p}=0,045)$. Авторы пришли к выводу, что в течение одного кода клинические исходы со стратегией SS II были связаны с улучшением клинических результатов по сравнению с ЧKВ, выполненным у сопоставимых пациентов из исторической ким образом, работа жерденной команды» на основании шкалы риска SS II показала себя достаточно э. олее безопасной по сравнению с SS [16].

В последнее время в доступной литературе уже появиись нублиации результатов работ с применением шкалы риска SS II, указыва

Так, С. Саmpos и соавт. в своей работе проспективно оценили согласованность между решением «сердечно команды» и рекомендацией шкалы SS II относительно стратегии реваскуляризации миокарда у пациентов с трехсосудистым поражением коронарных артерий. В исследовании всех включенных пациентов направляли на ЧКВ, когда SS II предсказывал риск смертности в пользу ЧК или когда прогнозы рисков были равны между ЧКВ и КШ Тем не менее «сердечная команда» могла не согласиться c рекомендацией SS II и рекомендовать метод реваскуля ризации в зависимости от тех или иных причин. В исследование всего были включены 178 пациентов, 167 (82,7\%) из которых были мужчинами. Возраст колебался от 59 до 73,3 года (в среднем 67 лет). «Сердечная команда» была солидарна с рекомендуемой стратегией реваскуляризации SS II у $152(85,4 \%)$ пациентов. У 25 пациентов «сердечна команда» приняла противоположное решение. Основна причина того, что «сердечная команда» отменила решение выполнять ЧКВ согласно SS II и рекомендовала КШ, был перспектива более полной реваскуляризации (21 из 25 пациентов) миокарда. Пациенты, которым было рекомендовано КШ, имели значительно больший анатомический показатель SYNTAX ( $\mathrm{p}=0,03)$ и более высокий прогнозируемый риск смертности для ЧКВ $(\mathrm{p}=0,04)$ по сравнению с прочими пациентами, которые были включены в исследование. Авторы пришли к выводу, что SS II является болес точным инструментом для руководства решениями о лечении пациентов с трехсосудистым поражением коронарных артерий. Также была доказана высокая согласованность между решениями «сердечной команды» и результатами расчета рисков при помощи SS II [17].

Тот же C. Campos и соавт. [18] проанализировали эффективность шкалы риска SS II у пациентов из рандомизиро-
ванного исследования EXCEL [18, 19]. Сам по себе EXCEL представляет собой проспективное рандомизированное многоцентровое исследование, целью которого было определение эффективности и безопасности ЧКВ с использованием эверолимус-выделяющих стентов по сравнению с КШ у пациентов с поражением незащишенного ствола ЛКА при низком и среднем показателе SS $(<33)$. После завершения набора пашиентов в исследование EXCEL авторами бы проспективно рассчитан показатель SS II во всех группах больных, направленных на КШ и ЧКВ. В результате 4-летние прогнозы смертности составили 8,5 и $10,5 \%$ ля ЧК ние прогнозы смертности составили 8,5 и $10,5 \%$ для ЧКВ $95 \%$ дИ $0,43-1,50)$. В группе с низким ( $\leqslant 22)$ - 
Рис. 4. График согласованности межау прогнозируемой и ожихаемой смертностью. Круги - значение, рассчитанное с помошью SS. Треугольники - значение, рассчитанное
с помошью SS II. Опрехеляется хорошая согласованность межму ожимаемой и предсказанной 4-летней смертностью межиу ожидаемой и прессказанной 4-летней смен
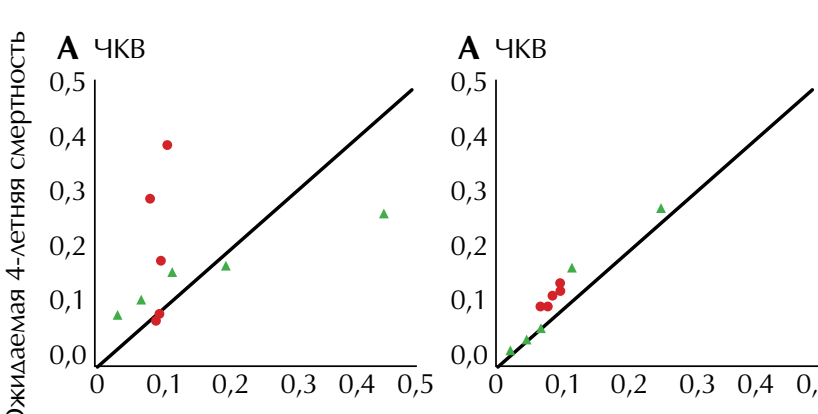

Прогнозируемая
4-летняя смертност

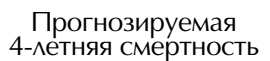

SS предсказанное ОШ было 0,69 (95\% ДИ 0,34-1,45); в промежуточных анатомических показателях SYNTAX (23-32) предсказанное ОШ было 0,93 (95\% ДИ 0,53-1,62). Авторы пришли к выводу, что показатель SS II указывает на равнозначный результат в долгосрочном прогнозе смерти между КШ и ЧКВ у пациентов с заболеванием незащищенного ствола ЛКА до среднего уровня риска согласно SYNTAX (SS<33). Как анатомические, так и клинические характеристики оказали явное влияние на прогноз смерти и принятие решений в пользу КШ или ЧКВ [19]

Крайне интересной представляется работа того же автора, выполненная на базе больных, включенных в многоцентровой регистр CREDO-Kyoto [20]. Целью исследования было оценить эффективность применения шкалы SS II в реальной Клинической практике с различными региональными и эпидемиологическими характеристиками пациенто из Японии. Для достижения поставленной цели была проанализирована смертность в отдаленном периоде у 3896 пациентов: у 2190 пациентов, перенесших ЧКВ, и у 1796 пациентов, перенесших КШ. SS II заметно различался в группах пациентов с КШ и ЧКВ (индекс конкордантности [c-index], 0,70, 95\% ДИ: $0,68-0,72,0,75,95 \%$ ДИ: 0,72-0,78). Шкала риска SS II показала большую согласованность между прогнозированной и ожидаемой смертностью у пациентов после ЧКВ или КШ (рис. 4). Авторы пришли к выводу, что SS II обладает надежной прогностической точностью ках в группе пациентов с КШ, так и в группах пациентов с ЧКВ и по сравнению с анатомическими SS более точна в стратификашии поздней смертности пашиентов при сложньх поражениях коронарных артерий [20].

Далее обратимся к еще одной публикации C. Campos и соавт., в которой авторы провели оценку SS II у 5433 пациентов после ЧКВ [21]. Целью данной работы была оценка клинической эффективности применения шкалы SS II при стратификации риска смерти в отдатенном периоле у пашиентов, которым были выполнены ЧКВ в совремод рандомизированных клинических исследованиях Для этого были проанализированы даннье из 7 современных РКИ

(5433 пациентов), в которых применялись стенты с лекар(5) Tirofb Sirling Bre-Mand Strolin

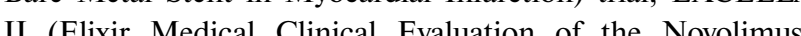
II Elixir Medical Clinical Evaluation of the NovolimusEluting Coronary Stent System) trial, LEADERS (Limus Eluted From a Duable Nersus Erodable Stent Coating) trial, MULTISTRATEGY (Multicenter Evaluation or Single HighDose Bolus Tirofiban Versus Abciximab With Sirolimus-Eluting Stent or Bare-Metal Stent in Acute Myocardial Infarction trial, RESOLUTE (Resolute All Comers) trial, исследование SIRTAX (Sirolimus-Eluting Stent Compared With PaclitaxelEluting Stent for Coronary Revascularization). Прогноз смерти после КШ или ЧКВ был рассчитан для каждого из пациентов. На основании прогноза шкалы SS II больные были разделены на три группы: пациенты, которым показано ЧКВ, пациенты, которым показано КШ, или те, которым может быть выполнено как ЧКВ, так и КШ (последнее означает равный прогноз между КШ и ЧКВ в долгосрочно перспективе согласно шкале SS II). В результате исследования по прогнозу SS II у $5115(94,14 \%)$ из 5433 пациентомогли быть выполнены как ЧКВ, так и КШ. У 271 больного согласно прогнозу SS II следовало выполнить только ЧКВ а у 47 пациентов - только КШ. Исходя из демографических и клинических характеристик пациентов надо отметить, что в группе больных, которым было рекомендовано и выполнено ЧКВ, пациенты были старше по возрасту, с более сохранной функцией ЛЖ, наличием ХОБЛ и значением SS ниже по сравнению с группой КШ. Пациенты, которым было рекомендовано выполнение КШ согласно шкале риска SS II (но было выполнено тем не менее ЧКВ), были моложе, в большей части мужчины, с более высоким значением SS, сниженной ФВ ЛЖ, и $17 \%$ имели атеросклеротическое поражение периферических артерий. При 3-летнем наблюдении по рекомендациям SS II пациенть, которым было рекомендовано выполнение КШ, имели более высокую смертность по сравнению с группами боль-

Рис. 5. Распреселение пашиентов после расчета SS и SS II. Есть у которых имеется расхожеение в прогнозе межиу SS и SS II [14]

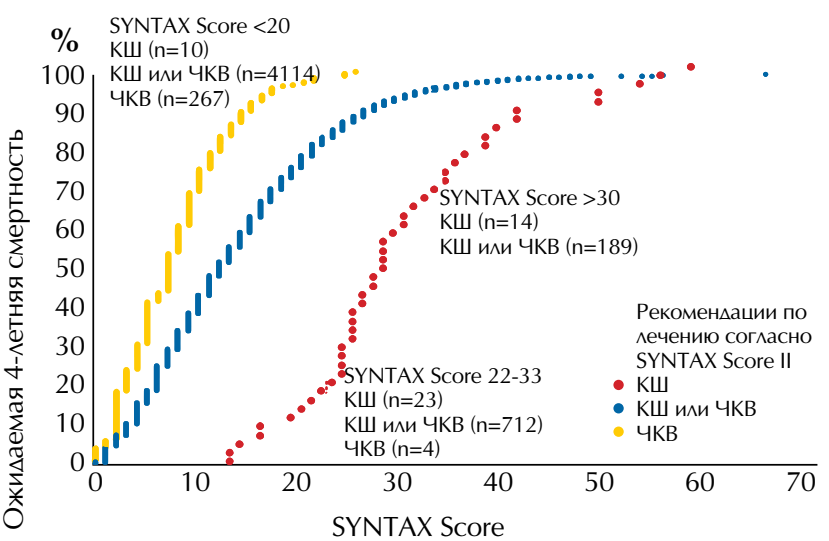

Рис. 6. ROC-анамиз SS и SS II при расчете риска смертности

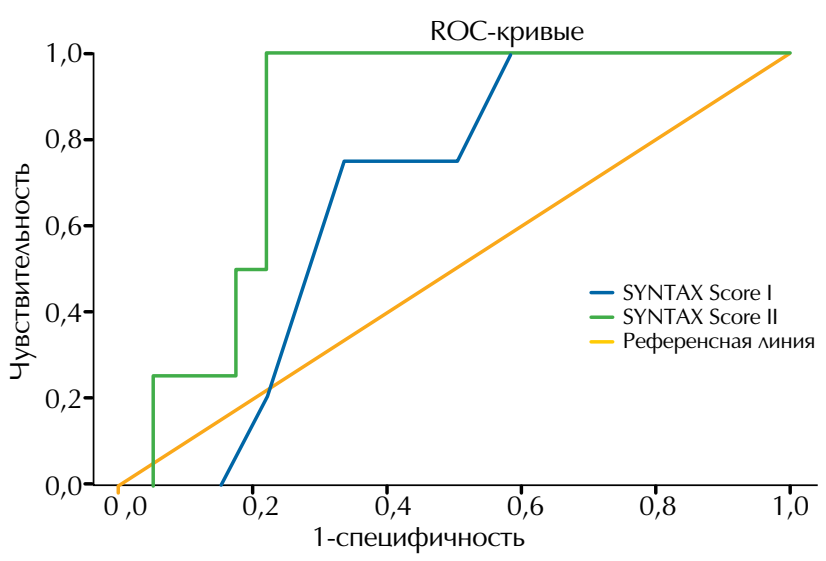

ных, которым было рекомендовано только ЧКВ или ЧК и КШ $(17,4,6,1$ и $5,3 \%$ соответственно, $\mathrm{p}=0,01)$. Одним и важнейших результатов исследования явилось то, что были выявлены пациенты, у которых значение SS указывало на необходимость выполнения KШ, но SS II определяла их группу возможного выполнения ЧКВ. Также имелись пациенты, у которых были показания к ЧКВ согласно SS, но SS II рекомендовала выполнение КШ (рис. 5). Данное обстоятельство позволяет формировать гипотезу о том, что повседневное использование SS II в работе «сердечной команды» позволит более точно и индивидуально выстраивать прогноз и выбирать более подходящий мето реваскуляризации, основанный как на анатомии, так и на сопутствующих патологических изменениях и прочих клинических характеристиках пациентов [21].

В 2017 г. А. Рillai и соавт. [22] исследовали отдаленные результаты после бифуркационного стентирования ствола ЛКА на основании шкал SS и SS II. Авторы проана изировали результаты бифуркационного стентирования 132 пациентов с изучением 4-летних отдаленных результатов 103 из них. Средний показатель SS среди пациентов составил $27,9+3,98$, а средний SS II $-29,43+9,005$. В результате было выявлено, что обшая частота больших карлиальных

Рис. 7. Прогнозируемая и фактическая 4-еетняя смертность у пашиентов поске ЧКВ и КШ. У пашиентов высокого риска
согласно шкале риска SS II при выполнении ЧКВ фактическая смертность оказалась выше прогнозируемой [23]

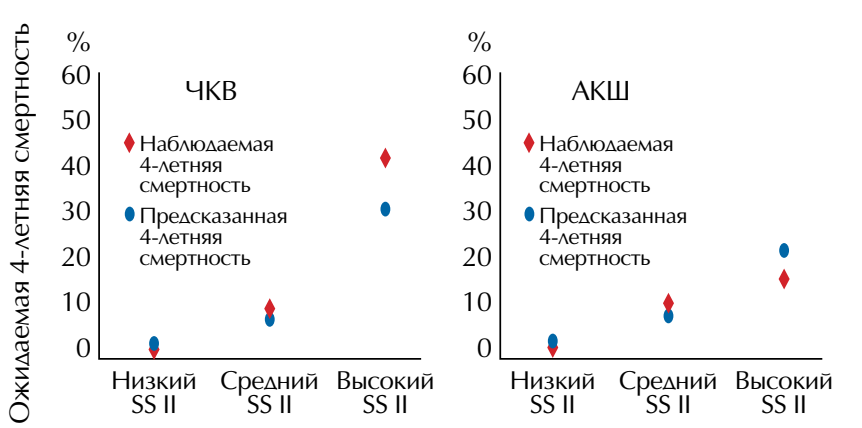

событий - повторная реваскуляризация, смерть, острый ИМ (ОИМ) или острое нарушение мозгового кровообра-
щения (ОНМК) - составила $17(16,5 \%)$ случаев. Частота повторных вмешательств была $11(10,7 \%)$. Также имели место 4 (3,9\%) случая смерти и 2 случая ОИМ. Не было выявлено ни одного случая ОНMK. Средние значения SS

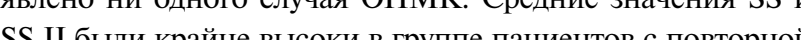

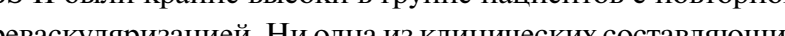

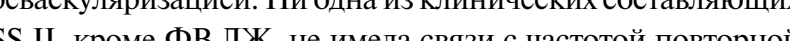
SS II, кроме ФВ ЛЖ, не имела связи с частотой повторно реваскуляризации. Интересно, что значение ФВ ЛЖ было значимо меньше в группе умерших пациентов $(\mathrm{p}=0,001)$ Средние значения SS и SS II были крайне высоки в группе пациентов со смертельным исходом, но без статистическо достоверности $(\mathrm{p}=0,087)$. Авторами также был проведе ROC-анализ для определения специфичности и чувствительности шкал. Площадь под ROC-кривыми для SS (как предиктор смерти) была равна 0,674, что составляет $75 \%$ чувствительности и $66,7 \%$ специфичности, с пороговым начением SS 29,5. В свою очередь, площадь под ROC-кривыми для SS II (как предиктор смерти) была равна 0,833, что составляет $100 \%$ чувствительности и $87,8 \%$ специфичности, c пороговым значением SS 34,35 (рис. 6). Таким образом, было доказано, что SS II является лучшим предикторо 4-летней смертности, чем его предшественник SS. Такж следует отметить, что ФВ ЛЖ является наиболее ценным показателем в SS II для прогнозирования отдаленных результатов после ЧКВ на стволе ЛКА [22].

N. Misumida и соавт. ретроспективно рассчитали SS II упциенов, перенесших ЧКВ или КШ. Авторы выяснили, что фактическая и прогнозируемая 4-летняя смертность хорошо коррелировала у пациентов с низким и средним риском по SS II в обоих когортах ЧКВ и КШ, но фактическая смертность была выше, чем прогнозируемая, у пациентов, подвергшихся ЧКВ с высоким риском по шкале SS II [23] (puc. 7)

M. Boukhris и соавт. исследовали применение шкаль SS II у пациентов с ОИМ и тяжелым трехсосудистым или стволовым поражением коронарных артерий. Следует отметить, что в SS II есть показатели, которые не могут измениться после выполнения реваскуляризации миокарда возраст, пол, атеросклероз периферических артерий, ХОБЛ. Другие показатели могут изменяться в результате выполненной реваскуляризации: клиренс креатинина, ФВ ЛЖ, анатомический показатель SS (то есть резидуальный SS), незащищенный ствол ЛКА. В исследование были включены 100 пациентов. Полная реваскуляризация миокарда была достигнута только у $2 / 3$ пациентов. У этих пациенто медиана резидуального значения SS составила 4. Далее авторы рассчитали остаточный SS II. Медиана этого показателя была равна 13. Интересно, что остаточный SS II $\geq 13$ был связан с развитием больших сердечных и цереброваскулярных событий в течение 1 года (коэффициент 1,93; 95\% ДИ: $1,18-6,81 ; p=0,037)$. Авторы пришли к выводу, что шкала SS II может быть использована для прогнозирования результаов первого года у пашиентов с ОИМ и тяжелым поражением коронарных артерий после выполненного ЧКВ [24].

Точная стратификация рисков у пациентов с тяжелы поражением коронарных артерий и сопутствуюшими за- 
болеваниями необходима не только для врачей, но и для карда. Известно, что различные современные клиниче(2центов. Необходимо оговаривать с больными и их родcher (c) осущеспій выбор меда ленения. Шкала SS 1, скорее sег, будег совер менее нам представляесся, что основная ее цель достигну-

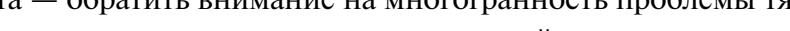
желого норажения коропарнах артерии и сонугспьющих им заболеваний, а также их взаимовлияние. Нередко пациент даже с крайне тяжелым поражением коронарных артерий согласно шкале SS будет имепь лучший прогноз и возможность лечения, если ему выполнить ЧКВ, и наоборог. Таким образом, на основании обзора доступной мировой литературы можно констатировать, что в настояще время медицинское сообщество работает в эпоху после проведенного исследования SYNTAX, на результаты которого опираются рекомендации по реваскуляризации мио-

ЛИTEPATYPA/REFERENCES

1. Marko Turina. The first PTCAs in Zurich, in 1977. European Heart Journal.
Volume 38. Issue 28. 21 July 2017. Pages 2166 -2167. Volume 38. Issue 28.21 July 2017. Pages 2166-2167.
Patrick W. Serruys, MD, PhD, Marie-Claude Morice, MD, A. Pieter Kappetein, MD, PhD, Antonio Colombo, MD, David R. Holmes, MD
Michael J. Mack. Percutaneous Coronary Intervention versus CoronaryArtery Bypass Grafting for Severe Coronary Artery Disease. $N$ Engl I Med

Authors/Task Force members, Windecker S., Kolh P., Alfonso F., Colle J. P., Cremer J., Juni P. 2014 ESC/EACTS guidelines on myocardia
revascularization: the Task Force on Myocardial Revascularization of the European Society of Cardiology (ESC) and the European -Thoracic Surgery (EACTS) developed with the special contribution of the European Association of Percutaneous
Cardiovascular Interventions (EAPCI). European heart journal. 2014 . 35(37), 2541-2619.

. U., Jüni P. ESC/EACT European heart journal. 2018 .

5. Campos C.M., Van Klaveren D., Faroog V., Simonton C.A., Kappetein

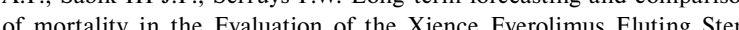
vs. Coronary Artery Bypass Surgery for Effectiveness of Left MaAn Revascularization (EXCEL) trial: prospective validation of the SYNTAX

Escaned J., Banning A., Farooq V., Echavarria-Pinto M., Onuma Y.
Ryan N., Suwannasom P. Rationale and design of the SYNTAX II trial evaluating the short to long-term outcomes of state-of-the-art

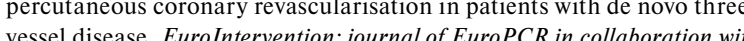
the Working Group on Interventional Cardiology of the European Society of Cardiology. 2016. 12(2), e224-34.

Dangas G.D., Farkouh M.E., Sleeper L.A., Yang M., Schoos M.M., Macaya insulin-treated diabetic patients. results from the FREEDOM trial Journal of the American College of Cardiology, 2014. 64(12), 1189-1197.

- AhnJ.M., RohJ.H.,., Kim Y.H. etal. Randomized Trial of Stents Versus Bypass Surgery for Left Main Coronary Artery Disease: 5-Year Outcomes of the

Morice M.C., Serruys P.W., Kappetein A.P., Feldman T.E. Stähle E., Colombo A., Religa G. Five-year outcomes in patients with left main disease treated with either percutaneous coronary yntervention or coronary artery bypass grafting
in the SYNTAX trial. Circulation. 2014 . CIRCULATIONAHA-113.

10. Iqbal J., Vergouwe Y., Bourantas C.V., Klaveren D.V., Zhang Y.J., Campos C.M., Steyerberg E.W. Predicting 3-year mortality after percutaneou coronary intervention: updated logistic clinical SYNTAX score based on Interventions. 2014. 7(5), 464-470. ияе и технические разработки в области эндоваскулярно хирургии приводят к достоверному улучшению результаов выполнения ЧКВ, особенно у пациентов со сложными поражениями коронарных артерий. Однако их совместное влияние на результаты ЧКВ недостаточно проанализировано. Как следствие вышеуказанного, результаты принятия решений «сердечной командой» на основании шкалы SS, вероятнее всего, не соответствуют реальной клинической практике и техническим возможностям эндоваскулярно хирургии. Это, в свою онередь, Приводит к сужению соременных возможностей эндоваскулярного лечения ИБС рий и сопутствующей хронической патологией

\section{Авторы заявляют об отсутствии конфликта интересов.}

11. Mohr F.W., Morice M.C., Kappetein A.P., Feldman T.E., Ståhle E., Colombo A., Houle V.M. Coronary artery bypass graft surgery versus and left main coronary disease: 5 -year follow-wn of the and let main coronary disease: 5 -year follow-up of the
clinical SYNTAX trial.

12. Алекян Б.Г., Стаферов А.В., Григорьян А.М., Карапетян Н.Г. Рентгенэндоваскулярная диагнностика и лечение заболевенаний сердца и сосудов в Российской Федера . . . №2. Стр. 93-240.
20. Петросян Ю.С., Иоселиани Д.Г. О суммарной оеннке состояния коронарного русла у больн
1976. №12. Стр. 41-46.

14. Vasim Faroo Serruys, MD, PhD. The SYNTAX Score and SYNTAX-Based Clinical Riss

Scores. Semin Thoracic Surg, 2011; 23:99-105.
Faroog V., van Klaveren D., Steyerberg E.W., Meliga E., Vergouwe Y., Chieffio making between coronary artery bypass surgery and percutaneous coronary intervention for individual patients: development and validation of SYNTAX .

C., Ryan N, Luigi De Maria G., Walsh S., Sabate M Hoole S.P. Clinical outcomes of state-of-the-art percutaneous coronary
revascularization in patients with de novo three vessel diseses: 1 -year results the SYNTAX II study. European heart journal. 2017. 38(42), , $1124-$-y 133 . .is
disease: Applying the SYNTAX S Score II in the Heart Team Discussion of the SYNTAX II trial. Catheteriza

Campos C.M., van Klaveren D., Faroog V., Simonton C.A., Kappetein A.P. Sabik IIIJ.F., Serruys P.W. Long-term forecasting and comparison of mortality in the Evaluation of the Xience Everolimus Eluting Stent vs. Coronary Artery (1.it) 2015. 36(20), $1231-1241$.

19. Genereux P., Serruys P., Kappetein A., van Boven A., Hickey M., Kandzari ANGIOGRAPHIC CORE LABORATORY ASSESSMENT. INSIGHTS FROM THE EXCEL TRIAL. Journal of the American College of Cardiology.

Klaveren D., Iqbal J., Onuma Y., Zhang Y.J., GarciaGarcia H.M., Nakagawa Y. Predictive performance of SYNTAX Score II in patients with left main and mu.
Journal. 2014. 78(8), 1942-1949.

21. Campos C.M., Garcia-Garcia H.M., van Klaveren D., Ishibashi Y., Cho Y.K
Valgimigigli M., Garg S. Validity of SYNTAX score II for risk stratification of D., POCock S. OUTCOMES OF ACI VERSUS CABG IN LEFT MAII 2017. 69(11 Supplement), 912. percutaneous coronary interventions: A patient-level pooled analysis of 5433 patients enrolled in contemporary coronary stent trials. International journal cardiology. $2015.187,111-115$.
Pillai A.A. Kottyath H.

Sesh S., Selvaraj R., Jayaraman B. Lonsterm outcomes following left main bifurcation stenting in Indian
population - Analysis based on SYNTAX I and II scores. Indian Hear population - Ant
Journal. 2017 .
23. Naoki Misumida at all. Prognostic Value of Anatomical SYNTAX Score and SYNTAX Score II in Veterans with Left Main and/or Three-Vessel Coronary Artery Disease. The American Journal of Cardiology. 201
Boukhris M. Abcha F., Tomasello SD Gibbilto S, Az

A R. Residual SYNTAX score II: A combination of, Azzarelli S., Galass revascularization degree and the clinical evaluation affer percutananeous coronary intervention. Journal of the Saudi Heart Association. 2018. 30(2), 158-159.

Поступила 31.012019 Принята в печать 04.03.2019 